\title{
Differential Polarization Laser Scanning Microscopy. Biological Applications
}

\author{
G. Steinbach ${ }^{* a}$, F. Besson ${ }^{\mathrm{a}}$, I. Pomozi ${ }^{\mathrm{b}}$, G. Garab ${ }^{\mathrm{a}}$ \\ ${ }^{a}$ Biological Research Center, Hungarian Academy of Sciences, Szeged, H-6701 Hungary \\ ${ }^{b}$ PI Vision Bt., Dagály u. 5., Budapest, H-1138 Hungary
}

\begin{abstract}
With the aid of a differential polarization (DP) apparatus, developed in our laboratory and attached to our laser scanning confocal microscope, we can measure the magnitude and spatial distribution of 8 different DP quantities: linear and circular dichroism (LD\&CD), linear and circular anisotropy of the emission (R and CPL, confocal), fluorescence detected dichroisms (FDLD\&FDCD, confocal), linear birefringence (LB), and the degree of polarization of fluorescence emission ( $\mathrm{P}$, confocal). The attachment uses high frequency modulation and subsequent demodulation, via lock-in amplifier, of the detected intensity values, and records and displays pixel-by-pixel the measured DP quantity. These microscopic DP data carry important physical information on the molecular architecture of anisotropically organized samples. Microscopic DP measurements are thought to be of particular importance in biology. In most biological samples anisotropy is difficult to determine with conventional, macroscopic DP measurements and microscopic variations are of special significance. In this paper, we describe the method of LB imaging. Using magnetically oriented isolated chloroplasts trapped in polyacrylamide gel, we demonstrate that LB can be determined with high sensitivity and good spatial resolution. Granal thylakoid membranes in edge-aligned orientation exhibited strong LB, with large variations in its sign and magnitude. In face-aligned position LB was considerably weaker, and tended to vanish when averaged for the whole image. The strong local variations are attributed to the inherent heterogeneity of the membranes, i.e. to their internal differentiation into multilamellar, stacked membranes (grana), and single thylakoids (stroma membranes). Further details and applications of our DP-LSM will be published elsewhere.
\end{abstract}

Keywords: confocal laser scanning microscopy, differential polarization, linear birefringence, thylakoid membrane, anisotropy

\section{INTRODUCTION}

Highly organized molecular macroaggregates are found in many hierarchically organized biological systems: DNAcondensates, cell walls, cytoskeletons, stacked membranes, membrane domains, protein aggregates etc. However, our understanding concerning their self-assembly, molecular organization, structural dynamics and physiological functions is still rudimentary [1]. Polarization spectroscopic techniques, and differential polarization spectroscopy in particular (e.g. linear and circular dichroism, LD and CD), provide important and unique information on this type of complex systems. In most cases, however, the measurements are difficult, or sometimes virtually impossible, to perform and/or interpret on macroscopic samples, and in their native environments. Hence it would be important to determine the microscopic DP parameters, and map the spatial distribution of these parameters of the anisotropic architecture.

The first differential polarization microscope, producing difference images generated with two orthogonal polarizations, was designed by Mickols et al. [2]. The DP microscope constructed by Juang et al. [3] was based on a scanning stage confocal microscope and used a DP attachment similar to those applied in modern dichrographs, i.e. a photoelastic modulator and a lock-in amplifier. It has been used to produce LD and CD images, with high sensitivity and high spatial resolution, on complex biological objects, such as chloroplasts and sickle cells [4,5]. The theoretical background of DP imaging has also been established and has been shown that different microscopic DP quantities provide important and unique information on the anisotropic molecular architecture of complex biological (or non-biological) samples [6,7]. Nevertheless, DP microscopic imaging is still in its infancy. This is partly due to the fact that the technique is far less elaborated and user-friendly than other scanning optical microscopic techniques, such as LSMs.

*stein@brc.hu; phone:+36-62-599600/478; fax:+36-62-433434 
A polarization-modulation laser-scanning microscope was constructed about a decade ago [8]. In this PM-LSM, the degree and direction of molecular alignment of rodlike molecules in Langmuir-Blodgett films could be imaged rapidly and sensitively through the magnitude and orientation of linear dichroism [9]. The operation of this DP microscope was confined to transmission regime, and thus did not allow confocal imaging. This might have impeded its use in biology. The DP-LSM designed and constructed by us [10], in addition to linear birefringence and linear dichroism, was made capable for the determination of additional differential polarization parameters, including fluorescence detected linear and circular dichroism (FDLD and FDCD), the linear and circular anisotropy ( $\mathrm{r}$ and CPL - circular polarized luminescence) as well as the degree of polarization of the fluorescence emission. These quantities, in contrast to LD and $\mathrm{LB}$, can be determined in confocal regime of the microscope and thus provide better resolution and image quality. Our main goal with the development of DP-LSM, which is suitable to measure virtually all DP quantities, was to provide an easy-to-use instrument for determining the most important anisotropy parameters of biological specimens. It must also be realized, however, that the significance of anisotropic organization in biology is not sufficiently well established. Thus, further work is needed on such biological samples in which anistropy is known to play structural/functional roles. Photosynthetic membranes belong to the few exceptions where the anisotropic molecular organizations of the pigment molecules and their (macro-) assemblies have been well established. In particular, it has been revealed that all pigment dipoles in all mature photosynthetic membranes are non-randomly oriented, and this anisotropy appears to have significant physiological roles [11,12]. Hence, photosynthetic membranes can be used to test the performance of the DPLSM and, at the same time, to further establish correlations between the known anisotropic parameters and microscopic DP parameters. In a recent publication we have shown that chloroplasts exhibit strong and inhomogenous LB, which, in the presence of linearly polarized laser tweezers, is large enough to lead to an efficient alignment, and thus its microscopic orientation can be manipulated [13]. We also pointed out that the direction of alignment is the same as that expected for non-birefringent disks $[14,15]$; i.e. the two mechanisms of orientation facilitate each other in a linearly polarized beam. However, large inhomogeneities and density variations, due to the presence of the stacked and unstacked membrane regions, and anomalous differential polarization scattering features, arising from the long-range order of the protein complexes, might hamper the expected rotation of this complex multilamellar membrane system in a circularly polarized beam. These special LB properties should be taken into account for a better understanding of the unusual properties of chloroplasts in a polarized beam of laser tweezers.

In this paper, using granal chloroplasts, we describe in more detail the method of LB imaging in our DP-LSM and demonstrate that LB imaging yields high resolution and high precision values, also amenable for statistical analyses, and thus might be a suitable tool in biological imaging. Additional examples of DP imaging, describing the technical details of the other DP imaging methods and further demonstrating the usefulness of DP imaging in biology, will be published elsewhere.

\subsection{Confocal microscopy}

Laser scanning microscope (LSM), when compared to the conventional microscopes, provide high quality and highresolution information on the structure of the specimen. The confocal principle is very useful to clean the image by cutting the out-focus-rays during the imaging. However, confocal imaging does not provide information on potentially very important parameters that are related to the anisotropic molecular architecture of the sample. In order to determine the anisotropy-related parameters polarization spectroscopy tools are required. Optimally, the two advantages, confocality and polarization spectroscopy should thus be combined. This can be achieved by using DP techniques combined with confocal laser scanning microscopy. By this means a more complete information can be obtained on the microscopic object, provided that the anisotropic molecular architecture exhibits large enough domains for microscopic observations. It must also be noted that confocal DP imaging, similarly to conventional LSM imaging, might be limited by the construction of the LSM, in our case a Zeiss LSM 410, which allows confocality only in fluorescence (and reflection) regime(s). 


\subsection{Differential polarization measurements}

The differential polarization quantities $\left(\mathrm{DP}=\left(\mathrm{I}_{1}-\mathrm{I}_{2}\right) /<\mathrm{I}>\right)$ can be calculated by measuring the intensity differences $\left(\Delta \mathrm{I}=\mathrm{I}_{1}-\mathrm{I}_{2}\right)$ between two orthogonal polarization states and $<\mathrm{I}>$, related to the total intensity; $<\mathrm{I}>=\mathrm{I}_{1}+\mathrm{I}_{2}$ or $<\mathrm{I}>=$ $\mathrm{I}_{1}+2 \mathrm{I}_{2}$, depending on the DP measurements. These orthogonal polarization states can be linear or circular, and they can be measured in the fluorescent light path (F) or in transmission (T). The measurements using the $\mathrm{F}$ beam path can be performed in confocal mode. Table I summarizes the DP quantities, their main technical parameters and physical information which can be obtained from the measurements.

\begin{tabular}{|c|c|c|c|c|}
\hline 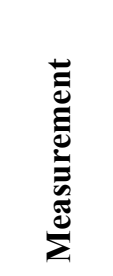 & 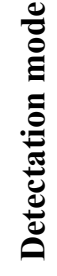 & 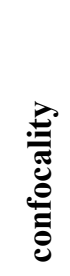 & 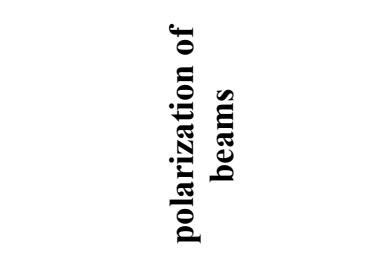 & Information on biological properties \\
\hline$P$ & $\mathrm{~F}$ & yes & $\begin{array}{c}\text { linearly polarized } \\
\text { excitation, fluorescence } \\
\text { intensity measurement }\end{array}$ & $\begin{array}{l}\text { microviscosity, fluorescence lifetime, } \\
\text { depolarization via energy transfer, } \\
\text { finding different domains }\end{array}$ \\
\hline$R$ & $\mathrm{~F}$ & yes & $\begin{array}{l}\text { unpolarized excitation, } \\
\text { analysis of linearly } \\
\text { polarised emission }\end{array}$ & $\begin{array}{l}\text { molecular architecture and anisotropic } \\
\text { organisation (emitting dipoles) }\end{array}$ \\
\hline$C P L$ & $\mathrm{~F}$ & yes & $\begin{array}{l}\text { unpolarized excitation, } \\
\text { analysis of circularly } \\
\text { polarized emission }\end{array}$ & $\begin{array}{l}\text { detection of chiral structures and minor } \\
\text { conformational changes (excited state) }\end{array}$ \\
\hline$L D$ & $\mathrm{~T}$ & No & $\begin{array}{l}\text { modulated linearly } \\
\text { polarized measuring beam }\end{array}$ & $\begin{array}{l}\text { molecular architecture and anisotropic } \\
\text { organisation (absorption dipoles) }\end{array}$ \\
\hline$C D$ & $\mathrm{~T}$ & No & $\begin{array}{l}\text { modulated circularly } \\
\text { polarized measuring beam }\end{array}$ & $\begin{array}{l}\text { detection of chiral structures and minor } \\
\text { conformational changes (absorption } \\
\text { dipoles) }\end{array}$ \\
\hline$F D L D$ & $\mathrm{~F}$ & yes & $\begin{array}{c}\text { modulated linearly } \\
\text { polarized excitation, } \\
\text { fluorescence intensity } \\
\text { measurement }\end{array}$ & $\begin{array}{l}\text { molecular architecture and anisotropic } \\
\text { organisation (fluorophores) }\end{array}$ \\
\hline$F D C D$ & $\mathrm{~F}$ & yes & $\begin{array}{c}\text { modulated circular } \\
\text { polarized excitation, } \\
\text { fluorescence intensity } \\
\text { measurement }\end{array}$ & $\begin{array}{l}\text { detection of chiral structures and minor } \\
\text { conformational changes (fluorophores) }\end{array}$ \\
\hline$L B$ & $\mathrm{~T}$ & No & $\begin{array}{l}\text { modulated linearly } \\
\text { polarized measuring } \\
\text { beams }\end{array}$ & $\begin{array}{l}\text { variations of the refractive index due to } \\
\text { anisotropic ultrastructure or texture }\end{array}$ \\
\hline
\end{tabular}

Table I: DP quantities, technical parameters and physical information from the measurements. 


\section{METHODOLOGY}

\subsection{Plant material and magnetic orientation of chloroplasts}

Chloroplasts were isolated from pea (Pisum sativum) leaves grown in the greenhouse by a method described earlier [16], and suspended in a reaction medium containing $30 \mathrm{mM}$ Tricine ( $\mathrm{pH}$ 7.6), $5 \mathrm{mM} \mathrm{MgCl} 2$ and $300 \mathrm{mM}$ sorbitol. Intact thylakoid membranes were oriented in a homogenous magnetic field of $1.5 \mathrm{~T}$, and trapped in the oriented state by inclusion in a polyacrylamide gel [4]. The preparation can be positioned in the magnetic field for edge-aligned (Figure 1) or face-aligned orientations (Figure 2). After $5 \mathrm{~min}$ the chloroplasts were oriented and fixed in the polymerized media.

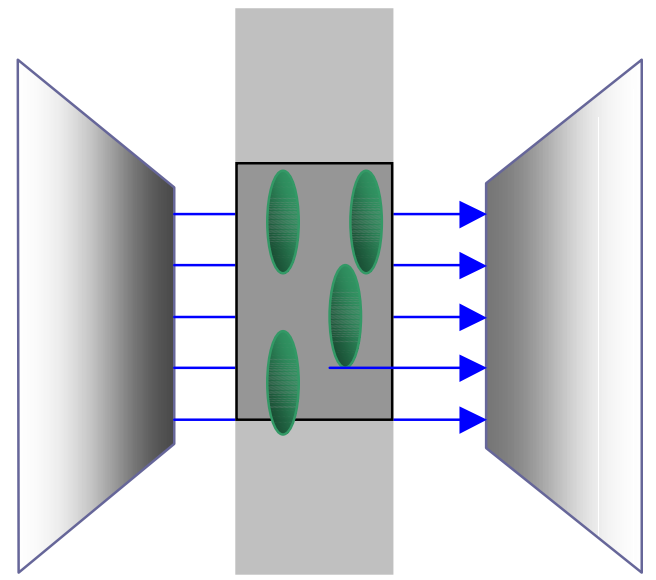

Figure 1: Edge-aligned chloroplasts (green) in a strong homogenous magnetic field (blue)

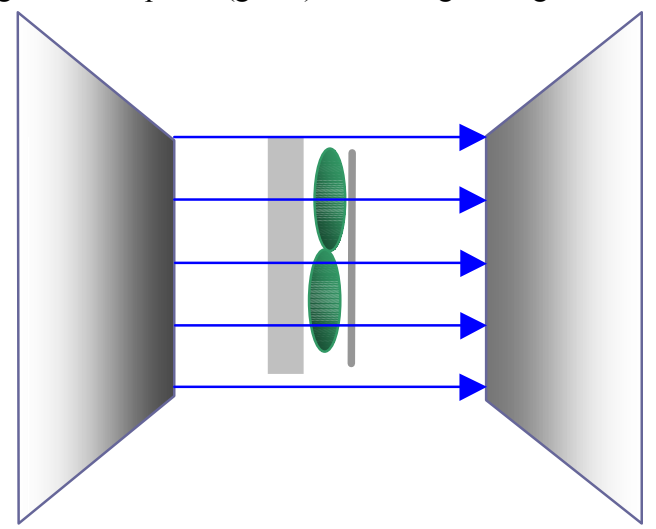

Figure 2: Face-aligned chloroplasts - the sample holder is rotated $90^{\circ}$ with respect to Figure 1

\subsection{Imaging of linear birefringence}

The scheme of the DP imaging set-up is shown in Figure 3A. A photoelastic modulator was placed between the linearly polarized laser source and the sample. The detector collected the transmitted light through the sample and an analyzer. The analyzer, placed between the sample and the detector was a linear polarization filter. A photoelastic modulator (PEM-90, HINDS Instruments) was used for the modulation of the polarization of the $543 \mathrm{~nm}$ HeNe laser beam (Figure 3B). For demodulation of the intensity signal a lock-in amplifier, a digital phase sensitive detector card was used (DPSD, KFKI, Budapest; maximum frequency: $400 \mathrm{kHz}$, minimum time constant: $100 \mu \mathrm{s}$ ), which was connected to a PC via ISA bus. The average intensity (DC component) of the signal was measured by an analogue low pass electrical circuit and by an analogue-digital converter (ADC) connected to the parallel port of the computer. The PC was an IBM compatible Pentium II computer with MS DOS operation system. The program was written in C environment, it was controlling the DPSD and ADC cards and was performing the on-line measurements and calculations. 


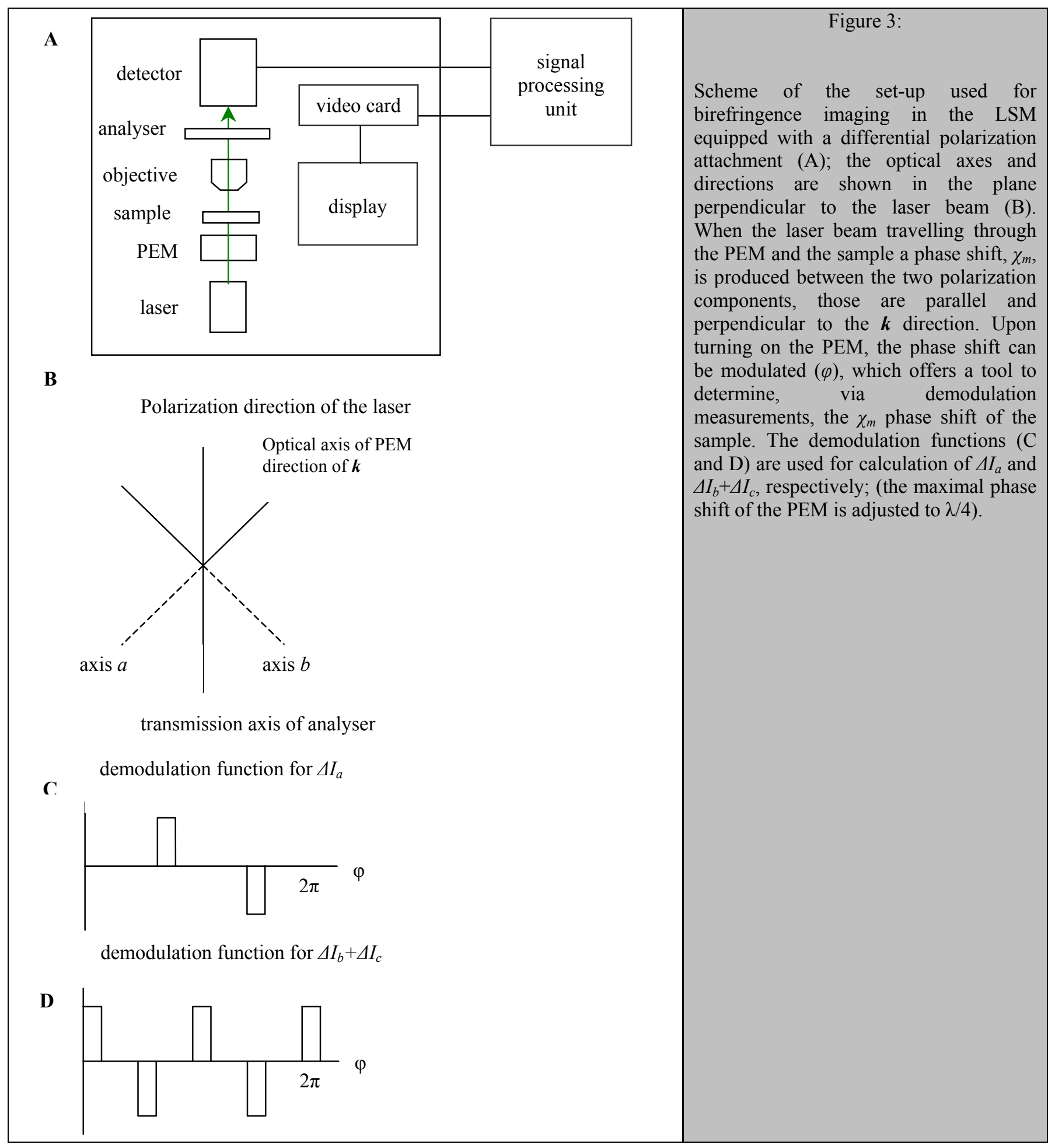


The optical phase shift with respect to a given $\mathrm{k}$ direction is the phase shift of the vibration of E-vector parallel and perpendicular to $\mathrm{k}$ while the light travels across the sample. The axis of birefringence for which the phase shift is maximal can be obtained by rotating the sample. In the case of our measurement on the chloroplasts the birefringence axis of the plastids was aligned at the $\mathrm{k}$ direction.

The optical phase shift, $\varphi$, between the directions parallel and perpendicular to $\boldsymbol{k}$ is varied periodically via the PEM. It is voltage controlled with a sinusoidal voltage signal, $U$, between $U_{\min }$ and $U_{\max }$ through $U_{0}=0$. After the sample the function of $\varphi$ is the following:

$\varphi=\chi+\varphi_{\max } \sin \left(\omega_{\text {mod }} t\right)$,

where $\omega_{\text {mod }}$ the frequency of PEM, $\varphi_{\max }$ is the phase shift of the PEM at maximal deflection (the amplitude of PEM), $\chi$ is the constant part of the phase shift including the persistent phase shift of the PEM (middle position, $U=U_{0}$ ), $\chi_{0}$, and the phase shift of the sample, $\chi_{m}, \chi=\chi_{0}+\chi_{m}$.

$\Delta I_{a}$ is the intensity difference measured between the maximal deviations of the PEM, $\Delta I_{b}$ and $\Delta I_{c}$ are the intensity differences measured between the middle and end positions of the PEM. Using $\Delta I_{a}, \Delta I_{b}$ and $\Delta I_{c}$ :

$S=\Delta I_{a} /\left(\Delta I_{b}+\Delta I_{c}\right)=\operatorname{tg} \chi \sin \varphi_{\max } /\left(1-\cos \varphi_{\max }\right)$

$\Delta I_{a}$ and $\Delta I_{b}+\Delta I_{c}$ are signals, which can be measured via demodulation; the demodulation functions are shown in Figure 3 $\mathrm{C}$ and $\mathrm{D}$.

Setting $\varphi_{\max }=\pi / 2$, the amplitude of PEM is equivalent to $\lambda / 4$ phase shift:

$S=\operatorname{tg} \chi$

The value of $\chi_{0}$ is measured without the sample, $\chi_{0}$ is usually function of the wavelength:

$S_{0}(\lambda)=\operatorname{tg} \chi_{0}(\lambda)$.

$\chi_{m}=\chi-\chi_{0}$.

\subsection{Image Analysis}

Images of the transmission intensity and of the linear birefringence of isolated chloroplasts can be obtained and stored in the computer associated with the microscope. Statistical analyses of the linear birefringence on chloroplasts were performed on 40 edge-aligned chloroplasts and 20 face-aligned chloroplasts. The average value, the distribution and the spatial repartition of the birefringence of edge- and face-aligned chloroplasts were calculated and compared using the software ImageJ.

\section{RESULT AND DISCUSSION}

\subsection{Main birefringence properties of the thylakoid membrane system}

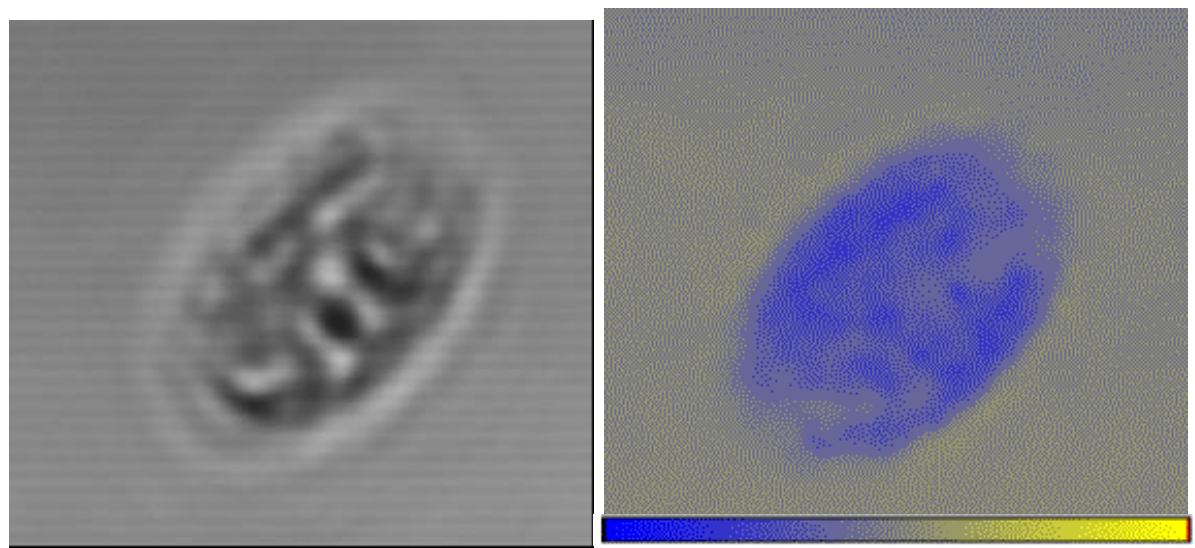

Figure 4: Transmission and linear birefringence images of an edge-aligned chloroplast. 


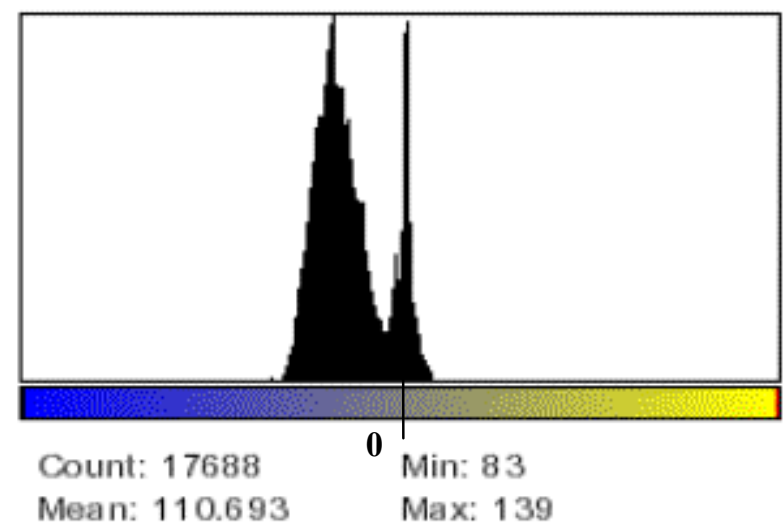

Figure 5.: Histogram of the birefringence values of the edge aligned chloroplast shown in Figure 4.

The basic linear birefringence features of isolated thylakoids can be illustrated on edge-aligned chloroplasts (Figure 4). It can be seen in the transmission image that the structure of chloroplasts is not homogenous. This is also reflected by their birefringence: the LB images exhibit strong inhomogeneities, which is depicted by using false colors.

\subsection{Statistical analysis}

In order to study more quantitatively the LB images, we performed different statistical analyses: histograms for entire images and distribution of phase shift values on selected areas of edge-aligned and face-aligned chloroplasts.

It can be seen in the histogram of the LB image (Figure 5) that edge-aligned chloroplasts exhibit two distinct sets of phase shift values: a set of strong positive and a set of weak negative values. These evidently do not average to zero, and thus the LB of edge-aligned chloroplasts do not tend to vanish.

As concerns the distribution of the phase shift values in different samples, we can see that, in good accordance with the histogram analysis, we find two sets of values. In each sample we selected 5 regions of $500 \mathrm{~nm}$ in diameter in blue regions and 5 in the yellow region (Figure 6). These measurements were processed on 40 edge-aligned chloroplasts, and plotted in Figure 7.

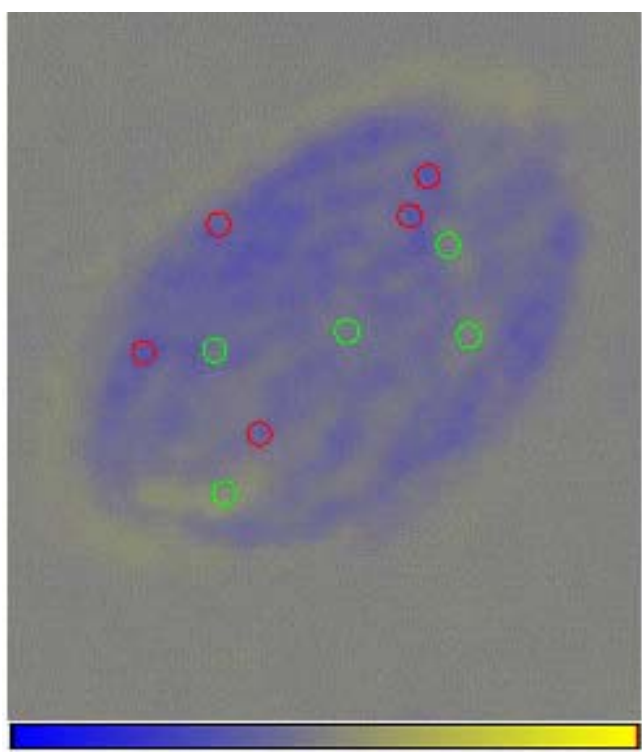

Figure 6: Birefringence (LB) image of an edge-aligned chloroplast. Shown in red and green, the measured regions used for calculating the values of the phase-shift in the sample. 
Repartition of phase shift magnitude

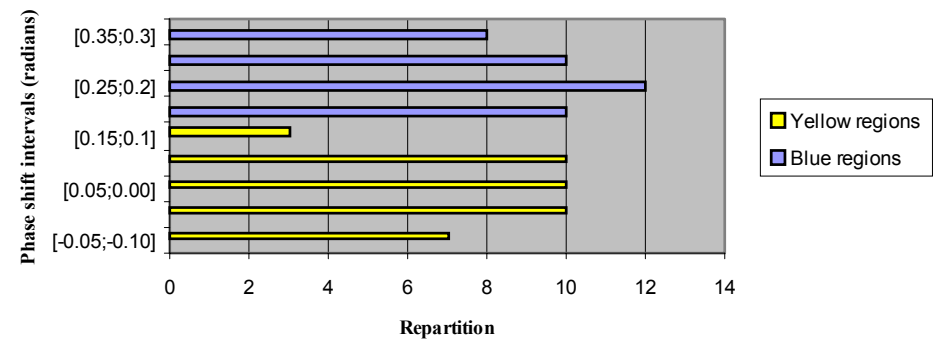

Figure 7: Phase shift repartition in edge-aligned chloroplasts in the blue and yellow regions as shown in Figure 6.

These data can be interpreted using the structure of thylakoid membrane in chloroplasts. It is reasonable to assume that the two distinct birefringent regions, which are co-existing in chloroplasts, originate from the laterally heterogeneous membrane system. The strong birefringence, with an average phase-shift value of 0.24 radians is assignable to grana, a stacked multilamellar membrane system. The much weaker LB values, averaging to a phase-shift of 0.01 radians can be assigned to the considerably less dense stroma thylakoids.

\subsection{Comparison of edge- and face aligned chloroplasts}

Imaging and statistical analyses were also performed on face-aligned chloroplasts (Figures 8 and 9). It can be seen that the birefringence is considerably weaker, the values are found in a much narrower interval than in the edge-aligned chloroplasts, and the blue and yellow regions overlap each other, and do not split into two sets.

These differences can be explained as follows. In edge-aligned orientation the membrane plane of the grana appears to define the preferential birefringence axis; in face-aligned orientation, because of symmetry, no preferential birefringence axis can be defined. (For imperfect orientation, and/or because of deviations from the idealized case, LB still occurs.) In line with this, in face-aligned orientation the anisotropy of the chloroplast is also much smaller than in the edge-aligned orientation, and tend to vanish in many samples.
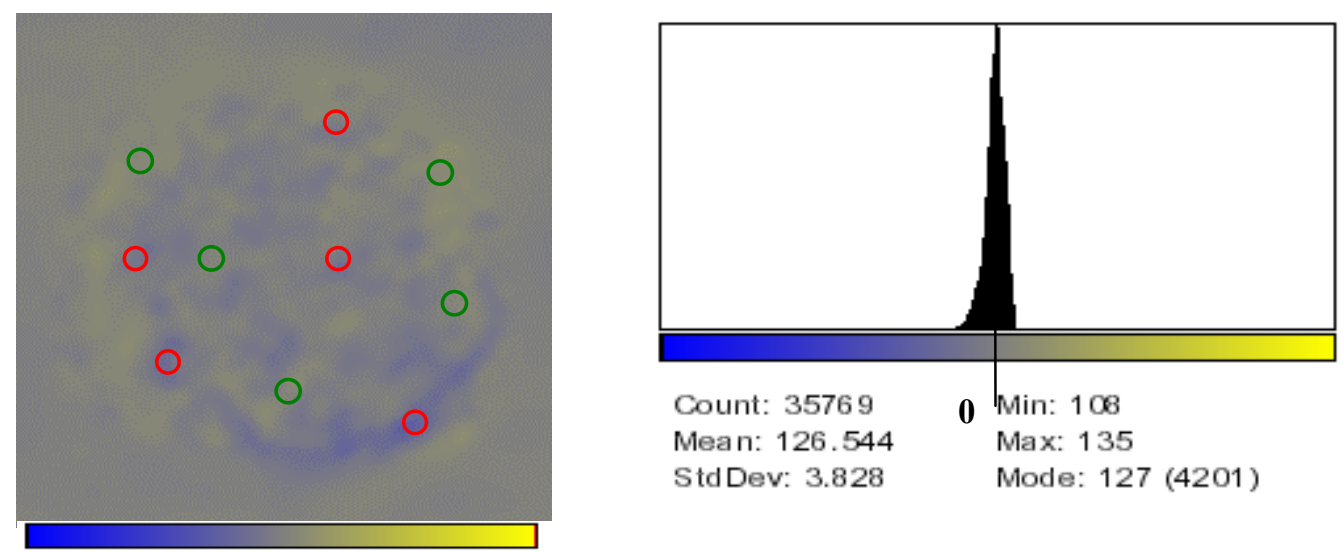

Figure 8: Birefringence (LB) image of a face-aligned chloroplast. Shown in red and green, the measured regions used for calculating the values of the phase-shift in the sample. B: Histogram of the birefringence values on the whole chloroplast. 


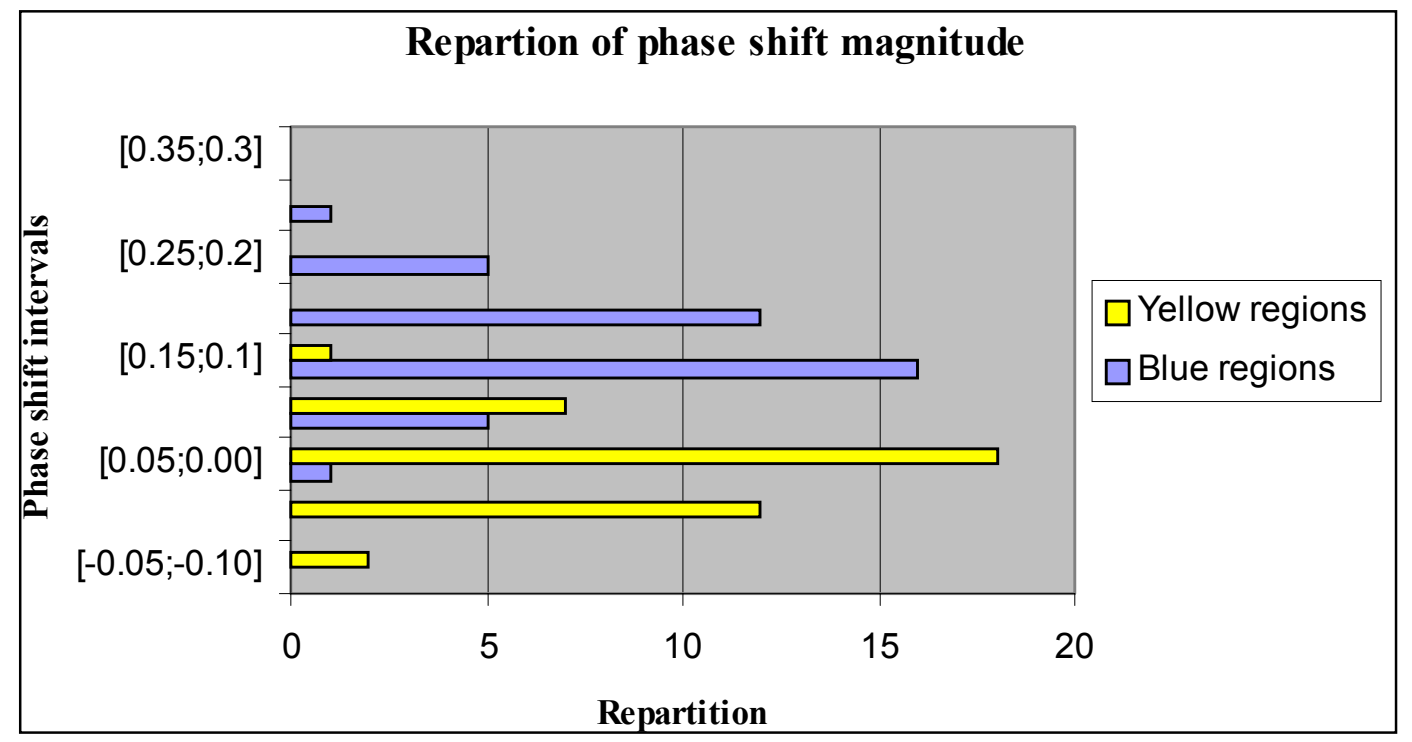

Figure 8: Repartition of the phase shift values in the preferentially blue and yellow regions.

\section{CONCLUSIONS}

We have determined the basic microscopic properties of chloroplasts. It is shown that granal thylakoid membranes exhibit strong but largely heterogeneous birefringence, which can be correlated with their internal membrane system. It is also shown, using thylakoid membranes aligned in a magnetic field and trapped in polyacrylamid gel, that the birefringence of face- and edge-aligned chloroplasts differ significantly from each other; this is explained by the symmetry of the membrane system, and is in agreement with other manifestations of the anisotropic molecular organization of the thylakoid membranes. Further studies, e.g. LB imaging on macro-assemblies of (light harvesting complexes, would probably allow us to make more quantitative assignments concerning the origin of strong LB in chloroplasts. Systematic studies on different biological samples should be done in order to establish the basic features and the structural-functional significance of the anisotropic organization in complex biological macroassemblies.

\section{ACKNOWLEDGEMENTS}

This work was supported by a grant from Hungarian Research Fund (OTKA 34188). The technical support by Dr. Georg Weiss and Dr. Reinhard Jörgens (Carl Zeiss Jena GmbH, Germany) in the construction of the differential polarization attachment to the Zeiss LSM 410 is gratefully acknowledged.

\section{REFERENCES}

1. Tinoco IJ, Mickols W, Maestre MF, Bustamante C: Absorption, scatering, and imaging of biomolecular structures with polarized light. Ann. Rev. Biophys. Biophys. Chem. 16: 319-349, 1987

2. Mickols W, Tinoco I, Katz JE, Maestre MF, Bustamante C: Imaging Differential Polarisation Microscope with Electronic Readout, Review of Scientific Instruments 56 (12): 2228-2236, 1985

3. Juang CB, Finzi L, Bustamante C: Design and Application of a Computer-Controlled Confocal Scanning Differential Polarization Microscope, Review of Scientific Instruments. 59 (11): 2399-2408, 1988

4. Finzi L, Bustamante C, Garab G, Juang CB: Direct observation of large chiral domains in chloroplast thylakoid membranes by differential polarization microscopy. Proc. Natl. Acad. Sci. USA 86: 8748-875, 1989

5. Beach DA, Bustamante C, Wells KS, Foucar KM: Differential Polarization Imaging . 3. Theory Confirmation Patterns of Polimerization oh Hemoglobin-s in Red Blood Sickle, Biophisical Journal 53 (3): 449-456,1988 
6. Kim M, Keller D, Bustamante C: Differential Polarization Imaging .1. Therory, Biophysical Journal 52 (6): 911927, 1987

7. Kim M, Bustamante C: Differential Polarization Imaging .4. Images in Higher Born Approximations, Biophysical Journal 59 (6): 1171-1182, 1991

8. Gupta VK, Kornfield JA: Polarization Modulation Laser-Scanning Microscopy - A Powerful Tool to Image Molecular Orientation and Order, Review of Scientific Instruments 65 (9): 2823-2828, 1994

9. Gupta VK, Kornfield JA, Ferencz A, Wenger G: Controlling Molecular Order in Hairy-rod Langmuir.blodgettfilms - a Polarisation-Modulation Microscopy Study, Science 265 (5174): 940-942,1994

10. Garab G, Pomozi I, Jörgens R, Weiss G: Method and apparatus for determining the polarization properties of light emitted, reflected or transmitted by a material using a laser scanning microscope, US Patent 6,856,391, 2005

11. Breton J, Vermeglio A: Orientation of photosynthetic pigments in vivo. In Photosynthesis. Govindjee, editor. Academic Press, New York. 153-93, 1982

12. Garab G: Linear and Circular Dichroism. In Biophysical Techniques in Photosynthesis. J.Amesz and A.Hoff, editors. Kluwer Academic Publishers, Amsterdam, 1996

13. Garab G, Galajda P, Pomozi I, Finzi L, Praznovszky T, Ormos P, Amerongen H: Alignment of biological microparticles by polarized laser beam, European Biophysics Journal with Biobhysics Letters 34 (4): 335-343, 2005

14. Galajda P, Ormos P: Orientation of flat particles in optical tweezers by linearly polarized light, Optics Express 11 (5): 446-451, 2003

15. Bayoudh S, Nieminen TA, Heckenberg NR, Rubinsztein-Dunlop H: Orientation of biological cells using planepolarized Gaussian beam optical tweezers, Journal of Modern Optics 50 (10): 1581-1590, 2003

16. Garab G, Faludi-Dániel A, Sutherland JC, Hind G: Macroorganization of chlorophyll a/b light-harvesting complex in thylakoids and aggregates: Information from circular differential scattering. Biochemistry 27: 2425-2430, 1988 